\title{
Neutrophil-to-lymphocyte ratio. Sixteen-year-long history since publication of our article in Bratislava Medical Journal
}

\author{
Zahorec R
}

Department of Anaesthesiology and Intensive Medicine, St. Elizabeth's Cancer Institute and Medical School of Comenius University, Bratislava. rzahorec@ousa.sk

Text in PDF www.elis.sk.

It is my pleasure to announce that a simple method of neutrophilto-lymphocyte ratio (NLR) represents an affordable, very sensitive and reliable inflammation and stress parameter nowadays broadly used in clinical practice all over the world. We defined the ratio of neutrophil count to lymphocyte count - now referred to as neutrophil-lymphocyte ratio for easy evaluation of inflammation, infection and sepsis intensity. In a cohort of 90 oncological adult patients, we have clearly proved that NLR correlates well with the intensity of inflammation and/or endocrine stress, and published our first results 16 years ago in Bratislava Medical Journal (Zahorec R.: Ratio of neutrophil to lymphocyte counts - rapid and simple parameter of systemic inflammation and stress in critically ill. (Bratisl Med $\mathrm{J} 2001 ; 102$ (1): 5-14). NLR is now an easily available biomarker that may be calculated from complete white blood cell (WBC) count differential and used for evaluation of low-grade (low intensity), medium-grade and high-grade (high intensity) inflammation. Some hospitals use NLR as a standard part of haemogram and white blood cell count differential (Bratislava, Prague, Netherlands).

The inflammation is a basic biologic and pathophysiologic process protecting the body; it requires activation of numerous component systems, including leukocytes, endothelium, thrombocytes, and multiple mediator networks. Inflammatory cells are the main driving force behind this process and comprise circulating leukocytes (populations of neutrophils, monocytes, and lymphocytes), in addition to fixed tissue macrophages, dendritic cells, mast cells and eosinophils (Bellingan, 1999). The haematologic system is a crucial organ for human immune defence, and plays a central role in inflammatory process and sepsis (Aird, 2003). The innate immune response together with acute phase response are highly evolutionarily conserved mechanisms that serve to combat pathogens, minimize tissue injury, and promote host recovery coupling with acquired immune response (Aird, 2003). NLR expresses a narrow relation between innate and adaptive cellular immune responses in health and disease. NLR is used as a subclinical inflammation

Department of Anaesthesiology and Intensive Medicine, St. Elizabeth's Cancer Institute and Medical School of Comenius University, Bratislava

Address for correspondence: R. Zahorec, MD, PhD, Department of Anaesthesiology and Intensive Medicine, Faculty of Medicine, Comenius University and St. Elizabeth's Cancer Institute, Heydukova 10, SK-812 50 Bratislava, Slovakia. marker, as well as for high grade inflammation in invasive infection and sepsis. In this minireview, I would like to present clinical evidence that there are many medical disciplines that may apply NLR within their daily routine for early diagnosis and stratification of inflammation of various origin and intensity, such as emergency and intensive medicine, oncology, surgery, acute cardiology, neurology, infectious diseases, and even psychiatry and dermatovenerology.

\section{Emergency and intensive medicine}

The relation between lymphopenia, neutrophilia and bacteraemia were extensively studied by Wyllie et al (2004). In their retrospective study measuring the white blood cell count differential and haemocultures in a cohort of 21,331 adult patients examined in Oxford Reddcliff Hospital emergency department, they found out that although lymphopenia proved to be a better indicator, both neutrophilia and lymphocytopenia were also associated with bacteraemia. Both neutrophilia and lymphopenia were better bacteraemia-predicting factors than the total white blood cell count (Wyllie et al, 2004). A notable progress in this field was achieved by C. de Jager et al (2010) when NLR was reviewed as a simple promising method to assess systemic inflammation and bacteraemia in acutely ill patients in emergency department. They retrospectively evaluated the ability of conventional infection markers such as C-reactive protein (CRP) and total WBC count with NLR and lymphocyte count to predict bacteraemia in adult patients with positive blood cultures and age-matched and gender-matched patients with negative blood cultures. Agroup of bacteraemia patients was showing significantly higher serum CRP, lower lymphocyte count ( 0.8 vs 1.2$)$ and higher NLR of 20.9 vs 13.2, p $<0.001$; the best cut-off value for NLCR to predict bacteraemia at emergency department was 10 (de Jager et al 2010). Loonen et al (2014), tested the ability of various biomarkers (CRP, PCT, suPAR and NRL) to predict sepsis and blood stream infection (BSI) in a group of patients within the cohort of acutely ill patients with suspected blood stream infection; in diagnosis, they used PCR SepsiTest for rapid detection of bacterial and fungal pathogens. ROC curves showed that PCT covered a higher area under the curve (AUC 0.806) than suPAR (AUC 0.79) and NLCR (AUC 0.77 ) to differentiate patients with positive blood cultures and proven BSI. They measured sensitivity, specificity and other parameters 
of biomarkers validity. An excellent validity for precise diagnosis of BSI was reached when a combination of NLCR 12 and PCT 2.0 $\mathrm{ng} / \mathrm{ml}$, or NLCR 10.0 and suPAR $6.2 \mathrm{ng} / \mathrm{ml}$ was used (Loonen et al, 2014). In brief, all biomarkers -PCT, suPAR and NLCR may be applied to differentiate between infectious and non-infectious SIRS in an emergency department. NLCR represents a cheap, available and easy-to-determine biomarker to pre-select suspected SIRS patients for molecular analysis besides blood cultures (Loonen et al, 2014). The differential leukocyte count and C-reactive protein levels were used to differentiate sepsis from non-infectious SIRS. A total of 2,777 patients admitted to the ICU of two centres in 7 years were evaluated retrospectively to test the efficacy of CRP and haemogram parameters as sepsis indicators (Gucyetmez and Atalan, 2016). Multivariate analysis confirmed that the likelihood of sepsis increased when biomarkers and APACHE II or SOFA score were used together. The best reached cut-off values: APACHE II higher than 13, SOFA score higher than 4, CRP $40 \mathrm{mg} / \mathrm{l}$, WBC higher than 12.0 and lymphopenia less than 0.45 , platelet count less than 150 thousand/ $\mu 1$, and NLCR higher than 14.2 (Gucyetmez, 2016). UK group led by J. Salciccioli performed an observational cohort study of unselected critically ill patients, using a large clinical database, and applied multivariate logistic regression to examine the association of NLR quartiles with 28-day mortality. Total of 5,056 patients were included in their study with 28-day mortality rate of $19 \%$ and median NLR of 8.9 (ranging from 5 to 16). A stepwise increase in mortality occurred with increasing quartiles of NLR. The highest mortality was in the 4th quartile of NLR, namely 16.2 when it reached $28 \%$. However, NLR was not associated with mortality in patients with sepsis. In conclusion, NLR is associated with outcomes in unselected critically ill patients except for septic patients (Salciccioli et al, 2015). On the contrary, Zhang et al (2016) evaluated diagnostic efficiency of NLR, procalcitonin and C-reactive protein to predict sepsis in 120 consecutive critically ill patients. Procalcitonin and NLR maintained a high level of diagnostic performance to predict sepsis with AUC of 0.829 for PCT and 0.718 for NLR. Both PCT and NLR maintained optimal diagnostic performance among the tested markers (Zhang et al, 2016). The problem that NLR is not working well as a sensitive inflammation marker in septic patient (Salciccioli ,2015; Gucyetmez, 2016) was explained partially by clinical observational study conducted by Riché et al (2016). In their cohort of 130 patients with septic shock, they observed that early death was associated with low median of NLR 6.2 (non-survivors) compared with higher NLR of 12.5 in survivors $(\mathrm{p}<0.0014)$. Low NLR $(<7)$ in patients with septic shock is a significant risk factor for early death. Low NLR may be influenced also by septic patients with leukopenia and neutropenia. Substantial daily increase in NLR in septic patients was typical for late death (Riché et al, 2016). The importance and prognostic value of NLR in 333 adult consecutive patients with sepsis was confirmed in an observational study by X. Liu et al (2016). NLR correlated well in this cohort with clinical stages of sepsis, severe sepsis and septic shock, and clinical severity measured by APACHE II score. Very high NLR values were clearly associated with bad outcome and mortality (Liu et al, 2016). Other researchers have also confirmed that very high NLR values may serve as a useful reliable mortality marker (Balta et al, 2014, Liu et al, 2016, Nakamura et al, 2016).

\section{NLR in oncology}

Walsh et al (2005) observed that NLR might be used as a survival prognostic parameter in oncological patients. NLR higher than 5 measured prior treatment were associated with bad outcome and survival in patients with colorectal cancer (Walsh et al, 2005). Since then, plenty of clinical epidemiologic studies with retrospective analysis provided strong evidence that haematologic markers, in particular neutrophil-to-lymphocyte ratio, reliably predict survival in patients with solid malignancy (Guthrie, 2013). Many groups have investigated prognostic value of NLR in a variety of tumours, and at differing stages of oncological disease associated with different cut-off values for NLR (Guthrie, 2016). Cancer progression and survival are multifactorial and host immune response are increasingly recognised to play a major role. The development and maintenance

Tab. 1. Cut-off of neutrophil-to-lymphocyte count ratio (NLR) for stratification and prognosis of different solid gastrointestinal malignancies.

\begin{tabular}{|c|c|c|c|c|c|c|c|}
\hline $\begin{array}{l}\text { Author, published } \\
\text { in year }\end{array}$ & City, country & Solid cancer & Trial design & $\begin{array}{c}\text { Number } \\
\text { of patients }\end{array}$ & Parameter & $\begin{array}{c}\text { HR } \\
\text { significance }\end{array}$ & $\begin{array}{c}\text { Statistical } \\
\text { significance }\end{array}$ \\
\hline Walsh, 2005 & Suffolk, UK & Colorect. C18.-20. & Retrosp. & 230 & NLR & $\mathrm{p}<0.001$ & yes \\
\hline Leitch, 2007 & Glasgow, UK & Colorect. C19-20 & Restrosp. & 233 & NLR & not valid & no \\
\hline Neal, 2011 & Leicester, UK & Colorect C18.-20. & Restrosp. & 202 & NLR & $2.02 \mathrm{p}<0.001$ & yes \\
\hline Halazun, 2007 & Leeds, UK & Colrect $\mathrm{C} 18, .20$ & Retrosp. & 440 & NLR & $2.26 \mathrm{p}<0.001$ & yes \\
\hline Gomez, 2008 & Leeds UK & Colorect C18-20, & Retrosp. & 501 & NLR & $1.3 \mathrm{p}<0.032$ & yes. low \\
\hline Hung, 2011 & Tao-Yuan Taiwan & Colorect C18-20 & Retrosp. & 1040 & NLR & $1.29 \mathrm{p}<0.0112$ & yes. low \\
\hline Ding, 2010 & Guang Dong China & Colorectal C19-20 & Retrosp. & 141 & NLR & $4.88 \mathrm{p}<0.003$ & yes. high \\
\hline Kwon, 2012 & Busan, Korea & Colorect C18. -20. & Retrosp. & 200 & NLR & - ns. & no \\
\hline Mallappa 2013 & Harrow, UK & Colorect C18-20. & Retrosp. & 297 & NLR & $1.81 \mathrm{p}<0.028$ & yes \\
\hline Chiang, 2012 & Linkou, Taiwan & Colorect C18.-20. & Retrosp. & 3731 & NLR & $1.31 \mathrm{p}<0.013$ & yes. \\
\hline Ubukata, 2010 & Tokyo, Japan & Gastric C16 & Prosp. & 157 & NLR+IFN/IL-4 & $5.78 \mathrm{p}<0.001$ & yes high \\
\hline Alzawa, 2011 & Kashiwa, Japan & Gastric C16. & Retrosp. & 262 & NLR & $2.21 \mathrm{p}<0.012$ & yes \\
\hline Jung, 2011 & Gwianggiu, Korea & gastric $\mathrm{C} 16$. & Retrosp. & 293 & NLR a T-stage & $1.65 \mathrm{p}<0.019$ & yes \\
\hline Rashid, 2010 & Derby, UK & Oesophag C15. & Retrosp. & 294 & NLR & - ns. & no \\
\hline Wang, 2012 & Guangzhou, China & Gastric C16. & Retrosp. & 324 & NLR a GPS & $2.32 \mathrm{p}<0.014$ & yes \\
\hline
\end{tabular}

Expl. HR -hazard ratio, NLR neutropil-to lymphocyte ratio 
Tab. 2. Normal values of NLR: median 1.8-2.2 (1.00-2.39) in adult population.

\begin{tabular}{|c|c|c|c|c|c|}
\hline $\begin{array}{l}\text { Parameter } \\
\text { WBC count } \\
\text { Neutrophils }\end{array}$ & $\begin{array}{c}\text { Normal Values } \\
\text { of NLR }\end{array}$ & $\begin{array}{l}\text { Low-grade } \\
\text { Inflammation }\end{array}$ & $\begin{array}{c}\text { Moderate } \\
\text { Inflammation }\end{array}$ & $\begin{array}{l}\text { High-intensity } \\
\text { Inflammation }\end{array}$ & $\begin{array}{l}\text { Critically High } \\
\text { Inflammation }\end{array}$ \\
\hline $\begin{array}{l}\text { Leukocytosis } \\
\text { neutrophilia }\end{array}$ & $1.8-2.2$ (median) & $3.0-6.99$ & $7.00-10.99$ & $11.00-22.99$ & 23.00 and higher \\
\hline $\begin{array}{l}\text { Leukopenia } \\
\text { neutropenia }\end{array}$ & $\begin{array}{c}1.00-2.39 \\
(5 \%-95 \%)\end{array}$ & $0.90-0.71$ & $0.71-0.4$ & $0.4-0.1$ & Less than 0.1 \\
\hline
\end{tabular}

NLR values of 2.4-2.99 and low NLR 1.0-0.91 usually mean a grey zone of subclinical inflammation

of a systemic inflammation has been consistently observed to confer a poorer outcome, even death of cancer patients (Roxburgh, 2014). Local and regional inflammation around and inside a solid tumour and presence of immunocompetent cells in immediate tumour microenvironment play an important role in determining cancer progression. Local inflammation was an important prognostic marker predicting both enhanced survival and good outcome (Klintrup et al, 2007). On the other hand, systemic inflammation induced by tumour growth may further support cancer spreading progression and is referred to as cancer-associated systemic inflammation(CASI), which is composed of a number of activities of many inflammatory cells like neutrophils, lymphocytes, platelets, macrophages and eosinophils together with plenty of cytokines, in particular IL-6, IL-8, IL-17, chemokines, and other mediators of inflammation (Motomura et al, 2014, Kantola et al, 2012). Of these components, only neutrophils, platelets, NLR, albumin and C-reactive protein showed to have an independent prognostic value when multivariate analysis was applied (Proctor et al, 2014). Meta-analysis and systematic review of inflammation-based score system showed that NLR represents a more consistent and independent prognostic parameter in patients with gastrointestinal malignancy, group of solid organ malignancies and more advanced stages, when cancer disease is established (Guthrie, 2013). NLR may reflect a more advanced stage of disease with potentially more aggressive tumour behaviour. Recent studies focused on the emerging role of pro-inflammatory cytokines in plasma of patients with elevated NLR (higher than 5) (Tab. 1). Motomura et al (2014) showed that elevated NLR values were associated with an increase in cytokine IL-17 and peritumoral macrophages activity. NLR may help clinicians to predict both response to treatment and value of treatment after one chemotherapy cycle. It has been presented in two clinical trials that post-treatment NLR normalisation was a significant factor while worse prognosis was recorded in patients not normalising their NLR values. Thus, NLR may be a reliable marker in oncology for early diagnosis, stratification of severity and cancer progression, monitoring of response to oncotherapy, and prognosis. As many authors have suggested, NLR maybe a key inflammation-based score parameter(Mohamed, 2014, Proctor, 2011, Pinato et al, 2014, Keong, 2016). NLR has supported evidence that tumour-associated inflammatory response had a paradoxical effect on enhancing tumorigenesis and progression (Colotta et al, 2009). Cancer-associated systemic inflammation can be considered core hallmark for cancer (Hanahan and Weinberg, 2011).

\section{Normal values of NLR}

NLR values are calculated by dividing the absolute count of neutrophils by absolute count of lymphocytes. Azab et al (2014) studied average NLR values in healthy adult population in USA; in total, 9,427 individuals were included in the study. The average values of 4,300 neutrophils/ $\mu 1$ divided by average value of 2,100 lymphocytes/ $\mu 1$ result in average NLR value of 2.15 . Yanti et al (2016) measured the average NLR of 1.95 in a cohort of 198 Indonesian young adults. Normal NLR physiologic ranges from 1.85 up to 2.15 (range $1.00-2.30$ ) depending on age and race. NLR values $<0.7$ and/or NLR higher than 2.5 should be assumed pathological; e.g. they provide evidence of low-grade inflammation in atherosclerosis, diabetes mellitus, immunosenescence and inflammation in old people. NLR may reflect the inflammation intensity with a simple rule that the higher the NLR value, the higher the inflammation intensity, namely latent sub-clinical (2.4-2.99), low-grade intensity inflammation (3.0-6.99), moderate inflammation (7.0-10.99), high intensity inflammation (11.0-22.99) and critically high NLR intensity (values exceeding 23.0). Low NLR values are presented in patients with severe sepsis and leukopenia/neutropenia, and or patients after chemotherapy Values below 0.7 are pathological, with moderate pathology below 0.4 and most severe inflammation even below 0.1 (Tab. 2).

\section{Conclusion}

Inflammation plays a major role in the pathophysiology of diseases commonly considered "non-inflammatory" such as cancer and atherosclerosis. Among many inflammatory markers, several studies demonstrated that elevated neutrophil-to-lymphocyte ratio (NLR) is a reliable and sensitive marker of inflammation and significant predictor of adverse outcome for patients with infection, sepsis, stroke, cardiovascular disease and cancer. We proposed clinical use of NLR to measure and objectively evaluate the intensity of inflammation and stress 16 years ago (Zahorec, 2001). More than 400 hundred papers were published since then, providing a large body of evidence that NLR can be used to evaluate inflammation, reflect the balance between innate and adaptive immune responses in many diseases and many medical disciplines (cardiology, oncology, intensive and emergency medicine). We suggested to use this parameter in routine clinical practice as a standard part of haemogram and white blood cell count differential.

\section{References}

May be provided by the author upon request

Received February 19, 2017. Accepted February 27, 2017. 